\title{
Eficacia y costo-utilidad de primer reemplazo total de cadera y rodilla en pacientes con osteoartritis
}

\author{
Carlos Rabago ${ }^{1}$, Christian Alfredo Waimann ${ }^{2}$, María Florencia Marengo ${ }^{2}$, \\ Julia Martínez ${ }^{2}$, Mario Menón ${ }^{2}$, Bruno Ivernizzi², Pedro Abatte ${ }^{2}$, Maximiliano Zuliani², \\ German Augusto Caputo², Juan Ignacio Chamorro², Néstor Fabián Pugliese ${ }^{2}$, \\ Nicolás Pietropaolo ${ }^{2}$, Fernando Eberle ${ }^{2}$
}

${ }^{1}$ Facultad de Ciencias Económicas de la UNICEN. ${ }^{2}$ Hospital Dr. Héctor Cura de Olavarría. Escuela Superior de Ciencias de la Salud de la UNICEN.

\section{R E S U M E N}

\section{Palabras clave:}

reemplazo total articular, eficacia, costo-utilidad, gonartrosis, coxartrosis, Argentina
Key words:

total joint replacement, efficacy, cost-utility, gonarthrosis, coxarthrosis, Argentine
Objetivo: Determinar la eficacia, costos y razón incremental de costo-utilidad (RICU) de la cirugía de primer reemplazo total de rodilla y cadera en pacientes con osteoartritis.

Material y métodos: Se diseñó un ensayo no controlado de tipo antes-después, incluyendo pacientes con osteoartritis primaria de cadera o rodilla en plan de primera cirugía de reemplazo articular total. El seguimiento incluyó los primeros 12 meses postcirugía, registrándose capacidad funcional [versión corta del Knee Injury and Osteoarthritis Outcome Score (KOOSPS) y Hip Disability and Osteoarthritis Outcome (HOOS-PS)], y calidad de vida (EUROQOL-5D). El análisis de costo se realizó desde la perspectiva de la sociedad, calculándose la eficacia, años de vida ajustados por calidad (AVAC), y RICU del procedimiento. Se definió un valor de p de 0,05 como estadísticamente significativo.

Resultados: Se incluyeron un total de 23 pacientes [coxartrosis $(52 \%)$, gonartrosis $(48 \%)$ ]. Los pacientes sometidos a reemplazo articular presentaron una mejoría estadísticamente significativa en la funcionalidad y calidad de vida de los pacientes [diferencia de medias: EU$\mathrm{ROQOL}=0,69, \mathrm{HOOS}-\mathrm{PS}=-49$, KOOS-PS=-31]. Al año de seguimiento se registró una ganancia de 0,43 AVACs. El costo de la cirugía y seguimiento postquirúrgico fue de 141.343 pesos por paciente, siendo el costo adicional de someterse al reemplazo de 3140 pesos por paciente. La RICU resultante fue de 7302 pesos por AVAC ganado.

Conclusiones: El reemplazo articular total resultó ser un procedimiento "muy costo-efectivo", mejorando la funcionalidad y calidad de vida de los pacientes con osteoartritis de cadera y rodilla.

\section{A B S T R A C T}

Objectives: To determine the efficacy, costs and incremental cost-utility ratio (ICER) of first hip and knee total replacement in patients with osteoarthritis.

Material y methods: We designed an uncontrolled before-after trial, including patients with primary hip and knee osteoarthritis waiting for first total joint replacement. Patients were followed for 12 months. Data collection included functional capacity [short version of Knee Injury and Osteoarthritis Outcome Score (KOOS-PS) and Hip Disability and Osteoarthritis Outcome (HOOS-PS)], and quality of life (EUROQOL-5D). Cost analysis were performed form societal perspective, determining efficacy, quality of life and life expectancy (QALY), and ICUR. A p-value of 0.05 was considered statistical significant.

Results: We included 23 patients [coxarthrosis (52\%), gonarthrosis (48\%)]. Patients showed a statistical significant improvement in functionality and quality of life one year after surgery [mean difference: EUROQOL=0.69, HOOS-PS=-49, KOOS-PS=-31]. Patient gained an average of 0.43 QALYs. The cost of surgery, including 12 months follow-up, was 141,343 Argentinean 
pesos (ARS), being the surgery incremental cost of 3140 ARS per patient. The ICUR was 7302 ARS per QALY.

Conclusion: The total joint replacement result to be a "highly cost-effective" procedure, improving functionality and quality of life in patients with knee and hip osteoarthritis.

Correspondencia

E-mail: christianwaimann@gmail.com

\section{Introducción}

El reemplazo total articular ha demostrado ser una intervención eficaz en pacientes con osteoartritis de rodilla y cadera no respondedores a terapia médica convencional ${ }^{1,2}$. Debido a esto, la frecuencia de su realización ha tenido una curva ascendente. En Estados Unidos, 515.400 reemplazos totales de rodilla fueron realizadas en el año 2007, significando esto un costo de 16 billones de dólares ${ }^{3}$. En 2009, el reemplazo total de rodilla se convirtió en el cuarto procedimiento más común en pacientes intrahospitalarios, habiéndose proyectado un crecimiento de cerca de 16 millones de procedimientos para el año $2030^{4,5}$. En el mismo país, en el año 2005 se realizaron 572.000 reemplazos totales de cadera, proyectándose un aumento de $174 \%$ para el año $2030^{4}$.

A pesar del gran impacto, solo unos pocos artículos han sido publicados evaluando la razón de costo-efectividad de estos procedimientos en comparación con el manejo médico no-quirúrgico de estos pacientes. En 2009, Losina y cols. desarrollaron un árbol de decisión para evaluar el costo-utilidad del reemplazo total de rodilla en pacientes con osteoartritis comparado con tratamiento no-quirúrgico. El procedimiento demostró ser una intervención costo-efectiva; sin embargo, la incertidumbre alrededor de la estimación fue amplia, variando entre $\approx \$ 9000 \mathrm{y} \approx \$ 107.000$ dólares por año de vida ajustado a calidad (AVAC) 6 . La población de este estudio solo incluyó mayores de 65 años, no consideró costos indirectos asociados y utilizó datos secundarios para el análisis. Waimann y cols. reportaron el costo-efectividad de reemplazo total de rodilla unilateral comparado con tratamiento médico ${ }^{7}$. Este estudio incluyó una cohorte de 250 pacientes pertenecientes a hospitales privados en Estados Unidos. El análisis se realizó desde la perspectiva de la sociedad, incluyéndose costos directos e indirectos. El procedimiento demostró ser efectivo en reducir el dolor y mejorar la capacidad funcional de los pacientes. La razón de costo-efectividad fue aceptable, requiriéndose $\$ 25.255$, \$35.274 y $\$ 56.908$ dólares para alcanzar una mejoría del $20 \%$, 50\% y $70 \%$, respectivamente.

En nuestro país, la frecuencia e impacto económico del uso de prótesis articulares es poco conocido. Entre el año 2004 y 2007, en un solo centro privado se reportaron 1180 reemplazos totales de cadera ${ }^{8}$. En el año 2009, Pastrián y cols. evaluaron el costo del reemplazo articular bilateral de rodilla en uno versus dos tiempos. Este análisis, incluyó solo costo hospitalario total, el cual fue en promedio de 37.159,69 pesos para quienes recibieron un reemplazo total de rodilla simultáneo, frente a $47.627,5$ pesos para quienes se sometieron al mismo procedimiento en dos tiempos ${ }^{9}$. Este análisis solo incluyó costos médicos directos, no realizándose evaluación de efectividad, ni comparación con tratamiento médico no-quirúrgico.

En la actualidad, no existe ningún estudio publicado que evalúe el costo-efectividad del reemplazo articular de cadera y rodilla en Argentina. En un reciente reporte realizado por la Federación Internacional de Seguros de Salud (IFHP, International Federation of Health Plans), el reemplazo de cadera y rodilla en Argentina presentó un costo hospitalario total de alrededor de \$3000 dólares por procedimiento, siendo el mismo significativamente menor que en países desarrollados como USA $(\approx 25.000$ dólares por procedimiento) ${ }^{10}$. Debido a estas discrepancias existentes en costos y utilización de servicios de salud, resulta incorrecta la extrapolación de datos de otros países, haciéndose necesario la realización de estudios a nivel local. En base a lo mencionado, nos planteamos los siguientes objetivos:

- Determinar la efectividad del primer reemplazo articular total para el tratamiento de la osteoartritis de cadera y rodilla. - Determinar los costos, razón de costo-utilidad de la cirugía de reemplazo total de rodilla y cadera en pacientes con osteoartritis.

\section{Material y métodos}

Se diseñó un ensayo no controlado de tipo antes-después. Se incluyeron pacientes con diagnóstico de osteoartritis primaria de cadera o rodilla en lista de espera para primera cirugía de reemplazo articular total. Como muestra se tomaron pacientes ambulatorios consecutivos que consultaron al servicio de Traumatología de un hospital público de la Provincia de Buenos Aries en el período enero a diciembre 2014. Los criterios de inclusión se detallan a continuación: (1) diagnóstico radiológico de osteoartritis primaria de cadera o rodilla; (2) estado cognitivo adecuado, determinado por el investigador; (3) habilidad para comunicarse en español. Se excluyeron pacientes con: (1) cirugía de revisión, (2) diagnóstico de artritis inflamatoria, (3) artropatía neuropática, (4) enfermedad de Paget u otra enfermedad ósea, (5) cirugía de reemplazo articular previa, (6) pacientes envueltos en un litigio legal relacionado con la cirugía.

El estudio fue aprobado por el comité de Docencia e Investigación del establecimiento y todos los pacientes firmaron el consentimiento informado previo a su participación en el estudio, conforme a las normas éticas de la Declaración de Helsinki de 1975.

El seguimiento incluyó una visita basal, en las dos semanas previas a la cirugía y una visita final, posterior al año del procedimiento quirúrgico. La información recolectada incluyó: datos sociodemográficos (edad, sexo, tiempo de evolución de los síntomas, actividad laboral, cobertura médica, y nivel educativo), clínicos, antropométricos, tratamientos previos y actuales. Además, los pacientes completaron diferentes cuestionarios, incluyendo:

Índice de comorbilidades de Charlson. Este cuestionario evalúa la presencia de 19 comorbilidades balanceadas de acuerdo a la morbilidad de las mismas, con un rango de 0 a 32, siendo 32 la mayor morbilidad. Se considera baja comorbilidad entre 0 y 1 puntos, comorbilidad moderada cuando el índice es 2 y alta comorbilidad cuando el valor es igual o superior a 3 puntos $^{11}$.

a. Índice Europeo de Calidad de Vida - EUROQOL - 5D (EQ - 5D) versión adaptada para la Argentina. Este cuestionario fue diseñado para evaluar la calidad de vida relacionada con la salud. Está constituido por 5 preguntas (movilidad, cuidadopersonal, actividades diarias, dolor/malestar, ansiedad/depresión) y una escala visual análoga de 0-100, donde 0 y 100 son el peor y mejor estado de salud, respectivamente ${ }^{12}$.

b. Knee Injury and Osteoarthritis Outcome Score - Physical Function Short Form (KOOS-PS): Autocuestionario derivado de 7-ítems pertenecientes al KOOS, que evalúa funcionalidad en pacientes con osteoartritis de rodilla ${ }^{13}$. Rango de $0-100$, donde 100 es la peor capacidad funcional.

c. Hip Disability and Osteoarthritis Outcome Score - Physical Function Short Form (HOOS-PS): Autocuestionario derivado de 7-ítems pertenecientes al HOOS, que evalúa funcionalidad en 
pacientes con osteoartritis de cadera ${ }^{13}$. Rango de 0-100, donde 100 es la peor capacidad funcional.

d. Productividad laboral: La pérdida de la productividad laboral se evaluó usando el cuestionario WPAI (Work Productivity and Activity Impairment). El WPAI evalúa: a) Ausentismo (tiempo de trabajo perdido); b) Presentismo: porcentaje de deterioro o efectividad en el trabajo; c) Pérdida de productividad total: combinación del ausentismo y presentismo; d) Deterioro de la actividad: porcentaje de deterioro experimentado en las actividades diarias.

\section{Análisis de costos}

Para el análisis primario se tomó en cuenta la perspectiva de la sociedad. El horizonte temporal será de un año posterior al reemplazo articular. No se aplicará descuento debido a la corta duración del seguimiento.

Se tuvieron en cuenta los siguientes $\operatorname{costos}^{15}$ :

- Costos directos médicos: internación relacionada a la cirugía de reemplazo, tipo de prótesis, honorarios correspondientes a cirujano y otros miembros del equipo médico, otras hospitalizaciones, rehabilitación, medicación, ortesis y ayudas marchas utilizadas, consultas ambulatorias, realización de procedimientos y estudios complementarios. Fuentes de información: entrevista estructurada, sistema informático hospitalario (registro electrónico de turnos, medicación y estudios complementarios). La asignación de los costos se actualizó a febrero 2016, de acuerdo al nomenclador y unidades básicas reportados por el Instituto de Obra Médico Asistencial de la Provincia de Buenos Aires (IOMA) ${ }^{16}$ y el nomenclador de prácticas de laboratorios de análisis clínicos sugerido por la Cámara de Instituciones de Diagnóstico Médico (CADIME) ${ }^{17}$. El costo de la medicación se asignó de acuerdo al vademécum nacional de medicamentos de Argentina reportado por la Agencia Nacional de Medicamentos, Alimentos y Tecnología Médica (ANMAT) ${ }^{18}$.

- Costos directos no médicos: Hace referencia al tiempo perdido (paciente y familiares) en recibir la asistencia médica, costo de desplazamientos no financiados por el sistema sanitario y costos de cuidadores contratados para apoyo de la enfermedad del paciente. Fuentes de información: entrevista estructurada. El costo del tiempo perdido se estimó de acuerdo al método del capital humano, multiplicando el tiempo perdido por el salario mínimo vital y móvil actualizado a febrero 2016, equivalente a 6060 pesos mensuales o 30,3 pesos por hora ${ }^{19}$. El cálculo de costos de transporte se realizó en base al tiempo de traslado reportado por los pacientes, aplicándole al mismo la fórmula desarrollada por Phibbs y Luft (1995): Distancia (millas $)=(($ Tiempo de transporte (minutes) $-3,42) / 1,70){ }^{*} 1,4^{20}$. La distancia obtenida se multiplicó por la tarifa vigente a febrero de 2016 para taxis/remises y colectivos. Para el caso de autos particulares se utilizó el Costo por Kilómetro de un Automóvil Mediano para uso profesional actualizado a febrero de 2016, valuado en 9,4 pesos por kilómetro ${ }^{21}$.

- Costos indirectos: Valoran los costos derivados de la ausencia del trabajo a causa de la enfermedad y de la pérdida de productividad como consecuencia de la osteoartritis de cadera y rodilla. Fuentes de información: entrevista estructurada, cuestionario WPAI ${ }^{22}$. Se calculó:

- Desempleo: se tomó la información a partir de la pregunta 1 del test WPAI.

- Horas perdidas de trabajo: se tomó la información a partir de la pregunta 3 y 4 del test WPAI.

- Días de baja: se tomó la información a partir de la pregunta 2 del test WPAI.

- Pérdida de productividad: se calculó en base a la pregunta 5 del cuestionario WPAI.

- Deterioro de la actividad: se calculó en base a la pregunta 6 del cuestionario WPAI.

El costo del tiempo perdido se calculó multiplicando el mismo por el salario mínimo vital y móvil actualizado a febrero 201619.

\section{Cálculo de razón incremental de costo-utilidad}

Para el análisis de costo-utilidad se utilizó un diseño de antes/ después, asumiendo que todos los cambios en calidad de vida y función son debidos a la cirugía de reemplazo articular. Como escenario alternativo (no realización del reemplazo articular) se asumirá una cohorte hipotética en donde los niveles basales de función, calidad de vida, tratamiento, prestaciones y costos permanezcan estables durante el período de seguimiento. Esta presunción se basa en reportes previos de cohortes de pacientes en lista de espera, en donde el dolor y la capacidad funcional no muestran un cambio significativo ${ }^{23-25}$. Se calcularon los costos y años de vida ajustados por calidad (AVAC), y se definió la razón incremental de costo-utilidad mediante la siguiente fórmula ${ }^{15}$ :

( $\Delta$ Costos en grupo cirugía - $\Delta$ Costos en grupo no-cirugía)

$$
\triangle \text { AVACs entre grupo cirugía y grupo no cirugía }
$$

Debido a no existir en la Argentina un valor establecido de disponibilidad a pagar ("willingness to pay") por AVAC ganado, se tomó como umbral la recomendación de la Comisión Macroeconómica de la OMS, siendo una intervención "muy costo-efectiva" si el costo es de hasta 1 Producto Bruto Interno (PBI) per cápita, "costo-efectivo" si el costo es de hasta 3 PBI per cápita, "no costoefectivo" si el costo es más de 3 PBI per cápita. Para este análisis, se tomó el valor del PBI per cápita 2015 (22.554 dólares), reportado por el Fondo Monetario Internacional ${ }^{26}$.

\section{Análisis de sensibilidad}

El impacto de la variabilidad de diferentes parámetros en la estimación de costos fue evaluado mediante análisis de múltiples vías (diagrama de tornado).

Los intervalos de confianza del 95\% (IC95\%) para costos y razones incrementales de costo-efectividad (RICE) fueron determinados mediante pruebas no paramétricas de bootstrapping con 3000 replicaciones, siendo el percentil 2,5 y 97,5, los límites inferior y superior de IC95\%, respectivamente.

Por último, se realizó un modelo alternativo de costo-utilidad, solo incluyendo costos directos médicos.

\section{Análisis estadístico}

Las características descriptivas de las variables cuantitativas se representan como media, desvío estándar e intervalo de confianza, mientras que las variables cualitativas se representan como porcentajes. Las variables cuantitativas se analizaron mediante test de la U de Mann-Whitney o t-test de Student, según correspondiese. El test de la $\chi 2$ (aplicando el estadístico exacto de Fisher cuando corresponde) se utilizó para las variables cualitativas. Para la comparación de los niveles basales y anuales de funcionalidad y calidad de vida, se utilizó t-test de Student pareado, Wilcoxon signrank test y test de chi-cuadrado de McNemar, según el tipo de variable y distribución de la muestra. Para el análisis de la capacidad funcional (KOOS-PS y HOOS-PS) se cuantifico la escala en forma continua y dicotómica. Debido a no existir punto de corte para estos autocuestionarios, se tomó como referencia otros cuestionarios funcionales utilizados en osteoartritis, los cuales definen un cambio clínicamente significativo de una mejoría absoluta de 20 puntos en una escala de 0 a $100^{\circ}$. Los datos faltantes fueron imputados utilizando la media de cambio en el resto de los pacientes, presentándose el análisis con o sin imputación de valores faltantes. Para el análisis estadístico se utilizó el paquete estadístico STATA 12. Se definió un valor de p de 0,05 como estadísticamente significativo.

\section{Resultados}

Descripción de la población - Características basales

En el período enero a diciembre de 2014, se reclutaron un total 
de 23 pacientes (porcentaje de aceptación del 100\%) con diagnóstico de coxartrosis $(52 \%)$ o gonartrosis $(48 \%)$ primaria en plan de primer reemplazo articular total. La media de edad fue de $70 \pm$ 16 años y 57\% eran de sexo masculino. Todos poseían cobertura médica. El nivel educativo en general era bajo: $41 \%$ primaria incompleta, $32 \%$ primaria completa, $14 \%$ secundario incompleto y $13 \%$ secundario completo. Del total de pacientes, un $32 \%$ vivía solo, $59 \%$ se encontraba casado/concubinato, $41 \%$ era soltero/viudo/separado. El 9\% eran tabaquistas activos y la mayoría poseía una baja morbilidad, presentando un índice de Charlson bajo en el $87 \%$ de los casos, moderado en el $9 \%$ y alto en $4 \%$. El peso medio fue de $78 \pm 4$, con un índice de masa corporal de $28 \pm 5$, encontrándose el $57 \%$ con sobrepeso, $14 \%$ obesos y $29 \%$ con peso adecuado.

\section{Eficacia de la primera cirugía de reemplazo articular de rodilla y cadera}

La Tabla 1 detalla la evaluación pre y postquirúrgica de los pacientes con osteoartritis terminal de cadera y rodilla. Basalmente, los pacientes presentaban niveles elevados de discapacidad funcional, con una reducción total de la capacidad física de 60 a $70 \%$. Los niveles basales de calidad de vida medida por EuroQoL eran bajos, con un valor de 0,47 sobre un máximo de 1 .

Del total de pacientes reclutados, un $83 \%$ completaron el seguimiento. Los cuatro pacientes que no concurrieron a la visita final, fueron contactados telefónicamente y se revisó su historia clínica electrónica, constatándose la ausencia de re-internaciones, muerte o complicaciones quirúrgicas. La media de seguimiento fue de $16 \pm 2$ meses. La Figura 1 detalla los cambios en la calidad de vida y funcionalidad desde la visita pre-cirugía hasta la última visita de seguimiento. Los pacientes sometidos a reemplazo articular presentaron una mejoría estadísticamente significativa en la funcionalidad y calidad de vida (Tabla 1), alcanzándose una mejoría clínicamente significativa en el $91 \%$ y $72 \%$ de los pacientes con reemplazo de cadera y rodilla, respectivamente. La mejoría global en la calidad de vida alcanzó al 91\% de los pacientes. Solo un paciente presentó un empeoramiento de la capacidad funcional al año de seguimiento. Además, se observó una mejoría en la capacidad de los pacientes para realizar actividades de la vida diaria medidas por WPAI, con una media final de $2 \pm 2$, lo que representó una mejoría de $5 \pm 3(\mathrm{p}<0,0001)$ para un valor máximo de 10 puntos (Figura 2).

Impacto económico de la osteoartritis de rodilla y cadera. Evaluación pre-quirúrgica

En concordancia con el grupo etario de mayor prevalencia de osteoartritis de rodilla y cadera, el $95 \%$ de los pacientes se encontraban jubilados y solo el $1(5 \%)$ pacientes se encontraba laboralmente activo, por lo cual no resultó posible calcular un valor representativo de desempleo, horas y días perdidos de trabajo, y
Figura 1. Limitación para la realización de las actividades de la vida diaria de acuerdo al WPAI (Work Productivity and Activity Impairment). Valores pre-quirúrgicos y en la visita final de seguimiento.

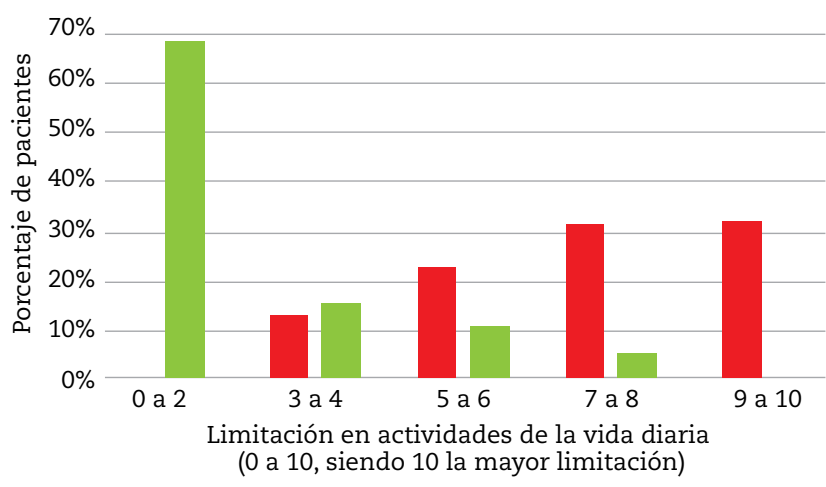

Vista basal Vista final

Figura 2. Distribución de costos asociados a reemplazo total articular incluyendo costos relacionados con la internación quirúrgica y seguimiento de los pacientes.

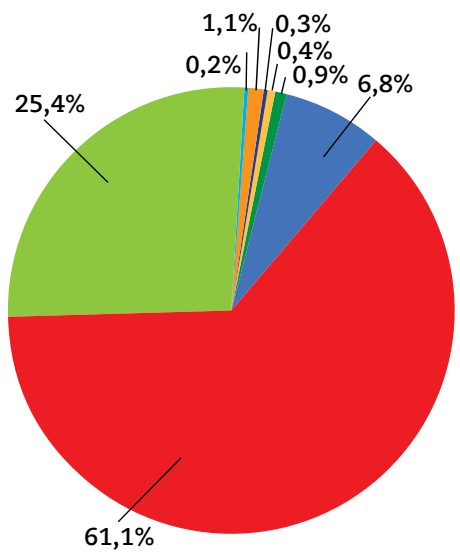

Atención

Ambulatoria

Rehabilitación

Exámenes

Medicación

Traslado

complementarios

Tiempo del

paciente

Tiempos de terceros

Prótesis e intervención quirúrgica

\section{Tabla 1. Eficacia de reemplazo de rodilla y cadera con y sin imputación de datos faltantes.}

\begin{tabular}{|c|c|c|c|c|c|}
\hline Variable & $\mathrm{n}$ & $\begin{array}{c}\text { Basal } \\
\text { Media } \pm \mathrm{DE}\end{array}$ & $\mathrm{n}$ & $\begin{array}{c}\text { Anual } \\
\text { Media } \pm \mathrm{DE}\end{array}$ & $\begin{array}{c}\text { Diferencia } \\
\text { Media (IC95\%), valor } \mathrm{p}\end{array}$ \\
\hline \multicolumn{6}{|l|}{ Calidad de vida } \\
\hline $\begin{array}{l}\text { EuroQoL } \\
\text { EuroQoL - con imputación }\end{array}$ & $\begin{array}{l}23 \\
23\end{array}$ & $\begin{array}{l}0,47 \pm 0,26 \\
0,47 \pm 0,26\end{array}$ & $\begin{array}{l}19 \\
23\end{array}$ & $\begin{array}{l}0,90 \pm 0,13 \\
0,90 \pm 0,12\end{array}$ & $\begin{array}{l}0,67(0,57 \text { a } 0,76), p=0,004^{*} \\
0,69(0,60 \text { a } 0,77), p<0,001^{*}\end{array}$ \\
\hline \multicolumn{6}{|l|}{ Discapacidad Funcional } \\
\hline $\begin{array}{l}\text { HOOS-PS } \\
\text { HOOS-PS - con imputación }\end{array}$ & $\begin{array}{l}12 \\
12\end{array}$ & $\begin{array}{l}61 \pm 18 \\
61 \pm 18\end{array}$ & $\begin{array}{l}11 \\
12\end{array}$ & $\begin{array}{l}12 \pm 13 \\
12 \pm 12\end{array}$ & $\begin{array}{l}-46(-33 a-59), p<0,00^{\&} \\
-49(-35 a-63), p<0,00^{\&}\end{array}$ \\
\hline $\begin{array}{l}\text { KOOS-PS } \\
\text { KOOS-PS - con imputación }\end{array}$ & $\begin{array}{l}11 \\
11\end{array}$ & $\begin{array}{l}64 \pm 21 \\
64 \pm 21\end{array}$ & $\begin{array}{c}8 \\
11\end{array}$ & $\begin{array}{l}37 \pm 25 \\
37 \pm 19\end{array}$ & $\begin{array}{l}-31(6 a-68), p=0,08 \&^{\$} \\
-31(-8 a-54), p=0,01^{\&}\end{array}$ \\
\hline
\end{tabular}

Abreviaturas: n, número de observaciones; DE, desvío estándar; KOOS-PS, Knee Injury and Osteoarthritis Outcome Score - Physical Function Short Form; HOOS-PS, Hip Disability and Osteoarthritis Outcome Score - Physical Function Short Form; EuroQoL-5D, Indice Europeo de Calidad de Vida de

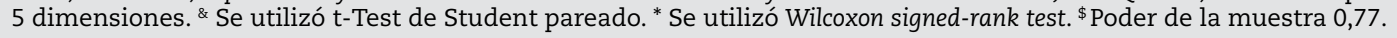




\section{Tabla 2. Costos médicos directos relacionados con la osteoartritis en los 3 meses previo a la visita basal.}

COSTOS MÉDICOS DIRECTOS relacionados con la osteoartritis en los últimos 3 meses

\begin{tabular}{|c|c|c|c|c|c|}
\hline \multicolumn{6}{|l|}{ Consultas médicas ambulatorias } \\
\hline & & \multirow[b]{2}{*}{$\mathrm{N}(\%)$} & \multicolumn{3}{|c|}{ Media $\pm \mathrm{DE}$ en total de pacientes } \\
\hline & & & Visitas & & Pesos \\
\hline $\begin{array}{l}\text { Visitas médicas } \\
\text { Rehabilitación (número de secciones) }\end{array}$ & & $\begin{array}{l}15(68 \%) \\
5(23 \%)\end{array}$ & $\begin{aligned} 1,6 & \pm 1,3 \\
3 & \pm 8\end{aligned}$ & & $\begin{array}{l}196,35 \pm 160,06 \\
166,20 \pm 455,60\end{array}$ \\
\hline \multicolumn{6}{|l|}{ Hospitalizaciones } \\
\hline & & & \multicolumn{3}{|c|}{ Media $\pm \mathrm{DE}$ en total de pacientes } \\
\hline & & $\mathrm{N}(\%)$ & Días estadía & & Pesos \\
\hline Hospitalizaciones relacionadas a la osteoartritis & & $0(0 \%)$ & ---o--- & & ------- \\
\hline \multicolumn{6}{|l|}{ Estudios complementarios ambulatorios } \\
\hline & & & Media $\pm \mathrm{DE}$ & & Pesos \\
\hline $\begin{array}{l}\text { Radiografías simples } \\
\text { Tomografía computada } \\
\text { Resonancia magnética } \\
\text { Ecografía musculo-esquelética }\end{array}$ & & & $\begin{array}{l}1 \pm 0 \\
0 \\
0 \\
0\end{array}$ & & $\begin{array}{c}204,47 \\
0 \\
0 \\
0\end{array}$ \\
\hline \multicolumn{6}{|l|}{ Medicación } \\
\hline & & & \multicolumn{3}{|c|}{ Media $\pm \mathrm{DE}$ en total de pacientes } \\
\hline & $\mathrm{N}(\%)$ & Dosis, mg/día & Mg/día & Pesos/mg & Pesos/día \\
\hline $\begin{array}{l}\text { Paracetamol } \\
\text { Opiáceos } \\
\text { Tramadol } \\
\text { Antiinflamatorios no esteroideos } \\
\text { Diclofenac } \\
\text { Ibuprofeno } \\
\text { Meloxicam } \\
\text { Ketorolac } \\
\text { Glucosamina + asociados } \\
\text { Omeprazol }\end{array}$ & $\begin{array}{r}2(1 \%) \\
1(<1 \%) \\
1(<1 \%) \\
18(82 \%) \\
9(41 \%) \\
4(18 \%) \\
4(18 \%) \\
1(<1 \%) \\
5(23 \%) \\
2(1 \%)\end{array}$ & $\begin{array}{l}2000 \pm 1400 \\
200 \pm 0 \\
84 \pm 35 \\
550 \pm 100 \\
15 \pm 0 \\
10 \pm 0 \\
1500 \pm 0 \\
20 \pm 0\end{array}$ & $\begin{aligned} 182 & \pm 664 \\
9 & \pm 43 \\
35 & \pm 47 \\
100 & \pm 220 \\
2 & \pm 5 \\
1 & \pm 2 \\
307 & \pm 597 \\
2 & \pm 6\end{aligned}$ & $\begin{array}{c}0,0009 \\
0,1568 \\
0,0290 \\
0,0006 \\
0,3638 \\
0,2843 \\
0,0026 \\
0,0742\end{array}$ & $\begin{array}{l}0,16 \pm 0,60 \\
1,43 \pm 6,69 \\
1,00 \pm 1,36 \\
0,06 \pm 0,13 \\
0,87 \pm 1,95 \\
0,13 \pm 0,61 \\
0,80 \pm 1,55 \\
0,13 \pm 0,44\end{array}$ \\
\hline
\end{tabular}

Abreviaturas: N, número de observaciones; Mg, miligramos; DE, desvío estándar.

\section{Tabla 3. Costos directos no médicos y costos indirectos relacionados con la osteoartritis en los 3 meses previo a la visita basal.}

\section{COSTOS DIRECTOS NO MÉDICOS}

Tiempo utilizado en atención médica por paciente y acompañantes

\begin{tabular}{|c|c|c|c|}
\hline & & \multicolumn{2}{|c|}{ Media $\pm \mathrm{DE}$ en total de pacientes } \\
\hline & Media $\pm \mathrm{DE}$ & Minutos & Pesos \\
\hline $\begin{array}{l}\text { Tiempo (min) utilizado en el traslado } \\
\text { Tiempo (min) utilizado en atención médica } \\
\text { Cantidad de acompañantes por visita }\end{array}$ & 0,5 & $\begin{array}{l}385 \pm 1006 \\
360 \pm 735 \\
452 \pm 1439\end{array}$ & $\begin{array}{l}195 \pm 508 \\
182 \pm 727 \\
228 \pm 727\end{array}$ \\
\hline \multicolumn{4}{|l|}{ Transporte no financiado por sistema sanitario } \\
\hline & & \multicolumn{2}{|c|}{ Media $\pm \mathrm{DE}$ en total de pacientes } \\
\hline & Media $\pm \mathrm{DE}$ & Kilómetros/ día & Pesos/día \\
\hline $\begin{array}{l}\text { Kilómetros por transporte propio } \\
\text { Kilómetros recorridos en remis } \\
\text { Kilómetros recorridos en colectivo }\end{array}$ & $\begin{array}{l}332 \pm 1249 \\
37 \pm 136 \\
139 \pm 573\end{array}$ & $\begin{array}{l}3,6 \pm 13,7 \\
0,4 \pm 1,7 \\
1,5 \pm 6,3\end{array}$ & $\begin{array}{l}34 \pm 128 \\
3 \pm 12 \\
2 \pm 6\end{array}$ \\
\hline \multicolumn{4}{|l|}{ COSTOS INDIRECTOS } \\
\hline & & \multicolumn{2}{|c|}{ Media $\pm \mathrm{DE}$ en total de pacientes } \\
\hline & $\mathrm{N}(\%)$ & Horas/día & Pesos/día \\
\hline $\begin{array}{l}\text { Cuidado a cargo de familiares o terceros } \\
\text { Pacientes empleados }\end{array}$ & $\begin{array}{c}11(50 \%) \\
1(5 \%)\end{array}$ & $7 \pm 11$ & $217 \pm 331$ \\
\hline Horas de baja laboral (pacientes) & -------- & $0,03 \pm 0,12$ & $0,8 \pm 3,7$ \\
\hline
\end{tabular}

Abreviaturas: Min, minutos; N, número de observaciones; DE, desvío estándar. 
Tabla 4. Costos médicos directos relacionados con la internación por cirugía de reemplazo articular, incluyendo exámenes pre-quirúrgicos.

\begin{tabular}{|c|c|c|c|c|}
\hline \multirow[b]{2}{*}{ Otros gastos } & \multirow[b]{2}{*}{ Pesos/día } & \multicolumn{3}{|c|}{ Media $\pm \mathrm{DE}$ en total de pacientes } \\
\hline & & Días de estac & & Pesos \\
\hline $\begin{array}{l}\text { Hotelería } \\
\text { Uso de material descartable }\end{array}$ & $\begin{array}{c}12,16 \\
3,2\end{array}$ & $3,0 \pm 0,5$ & & $\begin{array}{l}37,03 \pm 5,91 \\
9,75 \pm 1,55\end{array}$ \\
\hline Honorarios y gastos quirúrgicos & Pesos & $\mathrm{N}(\%)$ & & Pesos \\
\hline $\begin{array}{l}\text { Cirujano, Ayudantes, Anestesia y Gastos } \\
\text { Cirugía de Cadera } \\
\text { Cirugía de Rodilla }\end{array}$ & $\begin{array}{l}6521 \\
5034\end{array}$ & $\begin{array}{l}12(52 \%) \\
11(48 \%)\end{array}$ & & $5810 \pm 759$ \\
\hline Otras consultas médicas & Total & Número/dí & & Pesos \\
\hline $\begin{array}{l}\text { Cardiólogo } \\
\text { Kinesioterapia }\end{array}$ & $\begin{array}{l}1 \\
2\end{array}$ & - & & $\begin{array}{l}123,42 \\
142,34\end{array}$ \\
\hline Material quirúrgico - Prótesis & Pesos & $\mathrm{N}(\%)$ & & Pesos \\
\hline $\begin{array}{l}\text { Prótesis de cadera - Charley cementada } \\
\text { Prótesis de rodilla - Modular cementada }\end{array}$ & $\begin{array}{l}16900 \\
18000\end{array}$ & $\begin{array}{l}12(52 \%) \\
11(48 \%)\end{array}$ & & $17426 \pm 562$ \\
\hline Estudios complementarios & & Número determin & Iaciones & Pesos \\
\hline $\begin{array}{r}\text { Hemograma } \\
\text { Plaquetas } \\
\text { Coagulograma } \\
\text { Urea } \\
\text { Creatinina } \\
\text { Glucemia } \\
\\
\text { Electrocardiograma } \\
\text { Rx tórax frente }\end{array}$ & & $\begin{array}{l}2 \\
1 \\
1 \\
2 \\
2 \\
2 \\
1 \\
1\end{array}$ & & $\begin{array}{c}95,62 \\
26,19 \\
87,43 \\
62,90 \\
71,68 \\
58,02 \\
55,78 \\
108,18\end{array}$ \\
\hline Medicación & Total & ----/día & Pesos/ mg o ml & Pesos totales \\
\hline $\begin{array}{l}\text { Solución fisiológica isotónica por } 500 \mathrm{ml} \\
\text { Omeprazol cápsula por } 20 \mathrm{mg} \\
\text { Diclofenac ampollas por } 75 \mathrm{mg} \\
\text { Meperidina ampollas por } 0,1 \text { gramos } \\
\text { Cefazolina ampollas por } 1 \text { gramos } \\
\text { Enoxaparina } 40 \mathrm{Mg} \quad \text { Recibidos durante internación } \\
\text { Profilaxis postoperatoria (10 días) }\end{array}$ & 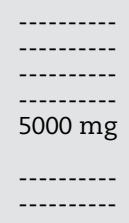 & $\begin{array}{c}3000 \mathrm{ml} \\
20 \mathrm{mg} \\
150 \mathrm{mg} \\
100 \mathrm{mg} \\
40 \mathrm{mg} \\
400 \mathrm{mg}\end{array}$ & $\begin{array}{l}0,0970 \\
0,0742 \\
0,2401 \\
0,4147 \\
0,0244 \\
6,3259 \\
6,3259\end{array}$ & $\begin{array}{c}886,23 \pm 141,35 \\
4,52 \pm 0,72 \\
109,68 \pm 17,49 \\
126,30 \pm 20,14 \\
122 \pm 0 \\
770,61 \pm 122,91 \\
2530,36 \pm 0\end{array}$ \\
\hline
\end{tabular}

Abreviaturas: N, número de observaciones; Mg, miligramos; DE, desvío estándar.

pérdida de productividad. Sin embargo, los pacientes presentaron un gran deterioro de su capacidad para realizar actividades de la vida diaria, con una media de deterioro de $7 \pm 2$, sobre un valor máximo de 10 puntos.

Las Tablas 2 y 3 detallan la utilización de recursos médicos y no médicos en los 3 meses previos a la cirugía de reemplazo articular, y los costos asociados a los mismos. Un paciente con osteoartritis terminal de cadera y rodilla, representa para la sociedad un gasto total de 8024 (IC95\% 3784 - 12.264) pesos por mes. El mayor porcentaje corresponde a costos indirectos [6953 (IC95\% $2642-11.264$ ) pesos/mes], seguido por costos directos no médicos [763 (IC95\% 560 - 965) pesos/mes] y costos médicos directos [329 (IC95\% 222 - 435) pesos/mes].

\section{Costos asociados a reemplazo total articular y seguimiento} postquirúrgico de los pacientes

La Tabla 4 detalla los gastos asociados al procedimiento quirúrgico. La media fue de 28.656 (IC95\% 28.513 - 28.798) pesos por paciente, correspondiendo la mayor proporción al material protésico (17.426 \pm 562 pesos), seguidos por los honorarios y gastos quirúrgicos (5810 \pm 759 pesos).

Las Tablas 5 y 6 describen los costos asociados al seguimiento postquirúrgico de los pacientes. El costo total fue de 112.687 (57.644 - 167.729) pesos. La necesidad de ayuda de terceros para cuidado personal y traslados representó el mayor porcentaje de los costos (Figura 2). El análisis de múltiples vías muestra que la variación en la utilización del tiempo de terceros representa el mayor impacto sobre los costos (Figura 3).

\section{Análisis costo-utilidad del reemplazo total de cadera y rodilla}

Para el análisis de costo-efectividad solo se incluyeron los pacientes que completaron el seguimiento (83\%) (Grupo intervención), comparándose con un grupo hipotético de pacientes no sometidos a cirugía (Grupo control). El costo adicional de someterse al procedimiento quirúrgico fue de 3140 (IC95\% -31.147 a 32.669) pesos por paciente. En $16 \%$ de los pacientes, el grupo intervención representó un ahorro para la sociedad con respecto al grupo control.

En cuanto a la eficacia, el grupo intervención presentó una ganancia de 0,43 (IC95\% 0,27 - 0,59) AVACs con respecto al grupo control.

En consecuencia, la razón incremental de costo-utilidad (RICU) para una primera cirugía de reemplazo articular total fue de 7302 (IC95\% -103.968 a 93.146) pesos por AVAC ganado.

En la Figura 4 se grafica la distribución de las muestras replicadas mediante bootstrapping en los diferentes cuadrantes de costo-efectividad. El $41 \%$ se encontraba en el cuadrante dominante (el procedimiento es efectivo y además representa un ahorro 
Figura 3. Diagrama de tornado evaluando la influencia de diferentes variables sobre los costos quirúrgicos $\mathbf{y}$ seguimiento de los pacientes.

\begin{tabular}{rr|l} 
Atención ambulatoria & 112.598 & 112.775 \\
Prótesis e intervención quirúrgica & 112.544 & 112.829 \\
Exámenes complementarios & 112.544 & 112.831 \\
Tiempo del paciente & 112.319 & 113.055 \\
Medicación & 112.248 & 113.174 \\
Rehabilitación & 112.206 & 113.168 \\
Traslado & 109.325 & 116.050 \\
Tiempo de terceros & & 164.148
\end{tabular}

$\$ 56.000 \$ 76.000 \$ 96.000 \$ 116.000 \$ 136.000 \$ 156.000 \$ 176.000$

Las barras representan el rango de costos cuando el valor del parámetro indicado varía desde el extremo menor al mayor del intervalo de confianza del 95\%.

para la sociedad) y el 59\% restante en el cuadrante de costo-efectividad (procedimiento efectivo, pero con costo adicional para la sociedad).

Utilizando como límite de disponibilidad a pagar el valor de hasta 1 Producto Bruto Interno (PBI) per cápita (22.554 dólares o 314.628 pesos), el $100 \%$ de las estimaciones se ubicaron por debajo de ese límite (nivel máximo de RICU estimado fue de 201.917).

En el modelo alternativo incluyendo solo costos médicos directos, el costo incremental de someterse al procedimiento quirúrgico fue de 28.025 (IC95\% 27.127 a 28.906) pesos por paciente, siendo la razón de costo-utilidad de 65.174 (IC95\% 47.369 a 106.197) pesos por AVAC ganado.

\section{Discusión}

Este estudio evalúa el impacto de la Osteoartritis (OA) terminal de rodilla y cadera, y la razón de costo-utilidad del reemplazo articular total en comparación con una estrategia hipotética no quirúrgica. En base a nuestro conocimiento, éste es el primer estudio que recolecta costos indirectos asociados al reemplazo articular de cadera y rodilla en Argentina. La osteoartritis de cadera y rodilla representa un deterioro multidimensional para la salud de los pacientes que la sufren, abarcando salud física, mental y desempeño en actividades sociales y de la vida diaria. En este grupo de pacientes, el reemplazo total articular resultó ser un procedimiento altamente efectivo, elevando significativamente la calidad de vida y capacidad funcional de los pacientes. Utilizando como cambio clínico significativo una mejoría de 20 puntos en una escala de 0 a 100, se observó una mejoría en 9 y 7 de cada 10 pacientes con reemplazo total de cadera y rodilla, respectivamente. Este límite de 20 puntos concuerda con una mejoría reportada por el paciente de "Algo mejor"27.

Desde una perspectiva de la sociedad, la razón de costo-utilidad de someterse al procedimiento quirúrgico fue de 7302 pesos por AVAC ganado. A pesar de la amplia variación en las estimaciones (IC95\% -103.968 a 93.146), todas se encontraron por debajo del límite de disponibilidad a pagar para un intervención "muy costo-efectiva" ${ }^{26}$. Desde la perspectiva del pagador de salud (solo incluyendo costos médicos directos), el procedimiento representó un costo adicional durante el primer año de seguimiento, presentó mínima variación entre los pacientes siendo aproximadamente de 28.000 pesos, resultando en una razón de costo-utilidad de aproximadamente 65.000 pesos por AVAC ganado, lo que corresponde a $20 \%$ del PBI per cápita, y representaría un procedimiento altamente costo-efectivo.

La utilización de estimaciones inferiores de PBI per cápita para el año 2015 (Banco Mundial: 13.467 USD), no afectó las con-
Figura 4. Análisis de sensibilidad. Distribución de las muestras replicadas mediante bootstrapping en los diferentes cuadrantes de costo-efectividad.

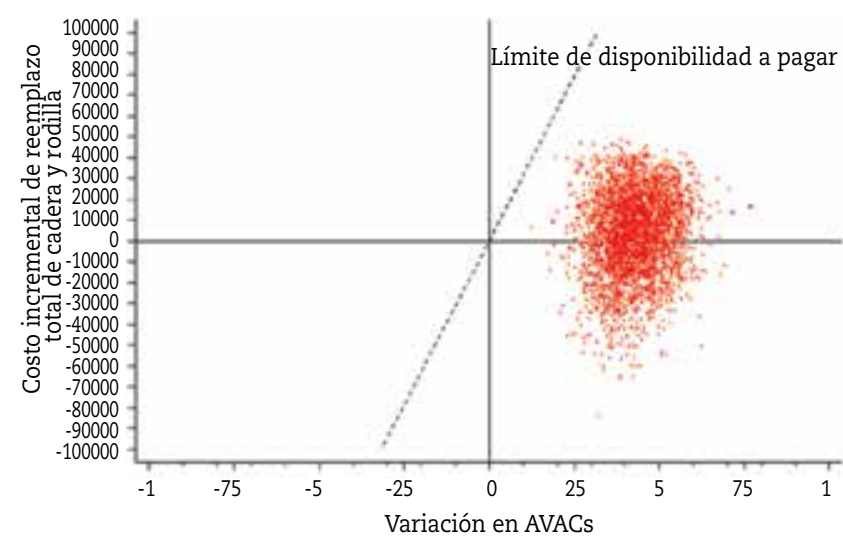

clusiones, ubicándose solo el 0,03\% de las estimaciones por encima del límite de disponibilidad a pagar, desde la perspectiva de la sociedad.

Es de resaltar, la diferencia de nuestras estimaciones con las provenientes de otros países ${ }^{7}$, lo cual concuerda con lo reportado por International Federation of Health Plans ${ }^{10}$. En comparación con Estados Unidos (costo aproximado de cirugía de 25.000 dólares), en la Argentina el costo incremental del procedimiento fue de 220 dólares (máximo 2300 dólares) y 1970 dólares (máximo 2035 dólares) por paciente, desde la perspectiva de la sociedad y el pagador de salud, respectivamente. Esto evidencia la necesidad de la realización de estudios locales para una correcta estimación de costo y utilidades.

Nuestra evaluación económica presenta diferentes fortalezas. Se desarrolla en la vida real, en el contexto del seguimiento y prácticas clínicas habituales, fuera de un protocolo de investigación previamente establecido. Por otro lado, presenta los costos y razones de costo-utilidad desde diferentes perspectivas, sociedad y pagador de salud, incluyendo la primera tanto de costos directos como de indirectos, ajustados a los valores de nuestro país.

Dentro del estudio también podemos enumerar ciertas limitaciones. Si bien consideramos que el método de elección para la evaluación de eficacia son los estudios clínicos randomizados, o en su defecto una cohorte alternativa en la cual no se lleve a cabo la intervención evaluada, esto no resulta posible en todos los casos. La osteoartritis terminal de cadera y rodilla genera un gran impacto en la calidad de vida, capacidad funcional y laboral de los pacientes, siendo el reemplazo articular la única alternativa en estos casos. A pesar de que en la literatura pueden encontrarse diferentes trabajos comparando tipos de prótesis o técnicas quirúrgicas, no existe ningún estudio randomizado que compare reemplazo articular versus tratamiento médico, siendo poco probable que esto alguna vez ocurra, por ser considerado un procedimiento ampliamente aceptado y con cobertura universal. Por otro lado, la inclusión de un grupo de control similar a los pacientes sometidos a cirugía sería muy difícil de lograr en la práctica. En general, aquellos pacientes que deciden no operarse, presentan mejor estado de salud, lo que introduciría un sesgo en el análisis. Por todo lo antes mencionado, pensamos que el uso de una cohorte hipotética de pacientes no sometidos a cirugía representa una modalidad válida de estimar el costo-efectividad de este procedimiento. Esta cohorte asume que el paciente continúa con el mismo esquema terapéutico y costos asociados previo a la cirugía, encontrándose el tratamiento de dichos pacientes a cargo del médico, no existiendo protocolo específico de tratamiento del dolor o rehabilitación kinésica.

Si bien, el tiempo de seguimiento elegido es relativamente corto para evaluar complicaciones a largo plazo, el plazo de un año resulta prudente para evaluar costos y eficacia, siendo el pe- 


\section{Tabla 5. Costos médicos directos y utilización de recursos relacionados con la osteoartritis posterior al reemplazo articular.}

COSTOS MÉDICOS DIRECTOS relacionados a la osteoartritis

Consultas médicas ambulatorias

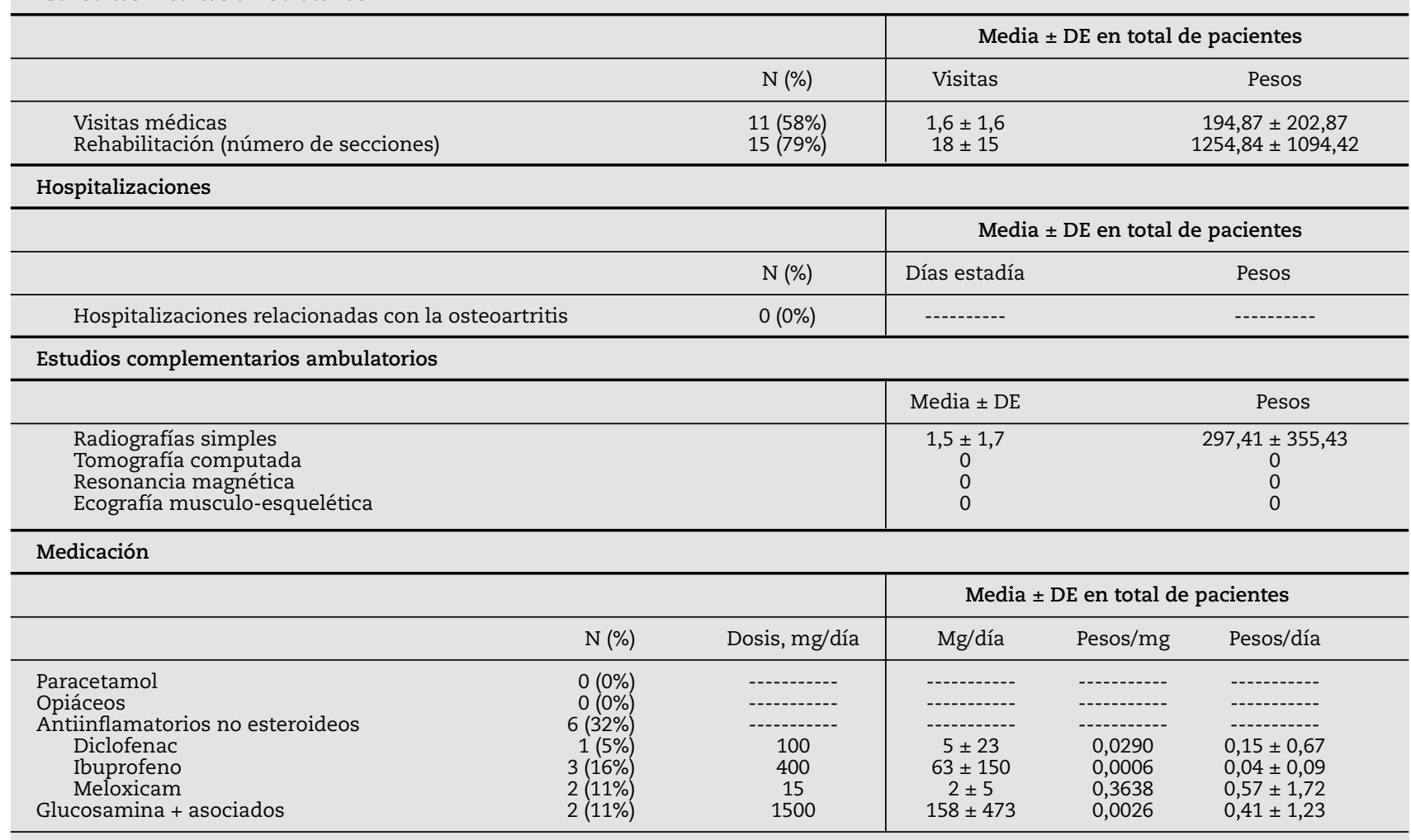

Abreviaturas: $\mathrm{N}$, número de observaciones; $\mathrm{Mg}$, miligramos; $\mathrm{DE}$, desvío estándar.

\section{Tabla 6. Costos directos no médicos y costos indirectos relacionados con la osteoartritis posterior al reemplazo articular.}

COSTOS DIRECTOS NO MÉDICOS

Tiempo utilizado en atención médica por paciente y acompañantes

\begin{tabular}{|c|c|c|c|}
\hline & & \multicolumn{2}{|c|}{ Media $\pm \mathrm{DE}$ en total de pacientes } \\
\hline & Media $\pm \mathrm{DE}$ & Minutos & Pesos \\
\hline $\begin{array}{l}\text { Tiempo (min) utilizado en el traslado } \\
\text { Tiempo (min) utilizado en atención médica } \\
\text { Cantidad de acompañantes por visita }\end{array}$ & $0,26 \pm 0,45$ & $\begin{array}{c}826 \pm 770 \\
1115 \pm 1026 \\
899 \pm 1769\end{array}$ & $\begin{array}{l}417 \pm 389 \\
563 \pm 518 \\
454 \pm 894\end{array}$ \\
\hline \multicolumn{4}{|l|}{ Transporte no financiado por sistema sanitario } \\
\hline & & \multicolumn{2}{|c|}{ Media $\pm \mathrm{DE}$ en total de pacientes } \\
\hline & Media $\pm \mathrm{DE}$ & Kilómetros/ día & Pesos/día \\
\hline $\begin{array}{l}\text { Kilómetros por transporte propio } \\
\text { Kilómetros recorridos en remis } \\
\text { Kilómetros recorridos en colectivo }\end{array}$ & $\begin{array}{l}543 \pm 640 \\
83 \pm 278 \\
200 \pm 682\end{array}$ & $\begin{array}{l}1,8 \pm 2,2 \\
0,2 \pm 0,8 \\
0,6 \pm 2,2\end{array}$ & $\begin{aligned} 17,4 & \pm 20,3 \\
1,5 & \pm 6,3 \\
0,6 & \pm 2,2\end{aligned}$ \\
\hline \multicolumn{4}{|l|}{ COSTOS INDIRECTOS } \\
\hline & & \multicolumn{2}{|c|}{ Media $\pm \mathrm{DE}$ en total de pacientes } \\
\hline & $\mathrm{N}(\%)$ & Horas/día & Pesos/día \\
\hline $\begin{array}{l}\text { Cuidado a cargo de familiares o terceros } \\
\text { Pacientes empleados } \\
\text { Horas de baja laboral (pacientes) }\end{array}$ & $\begin{array}{l}9(47 \%) \\
2(11 \%) \\
-\end{array}$ & $\begin{array}{l}6 \pm 10 \\
0 \pm 0\end{array}$ & $\begin{array}{c}174 \pm 296 \\
------ \\
0 \pm 0\end{array}$ \\
\hline
\end{tabular}

Abreviaturas: N, número de observaciones; Min, minutos; DE, desvío estándar. 
ríodo en donde se realizan la mayoría de los gastos asociados a la cirugía y se obtiene el pico máximo de mejoría clínica. El impacto de sobrevida de la prótesis y complicaciones a largo plazo están fuera del alcance de este análisis, ya que el seguimiento no supera el tiempo de latencia necesario para la aparición de estos eventos.

En cuanto a la asignación de costos, se debió utilizar diferentes fuentes por la falta de acceso a los costos crudos de cada recurso de salud. Esto se suma a la amplia variación de costos de los recursos tecnológicos e insumos utilizados (tipo de prótesis, técnicas quirúrgicas, etc.), contexto inflacionario y cambiario de nuestro país, lo que resulta en la necesidad de actualización y adecuación continua de los costos. Sin embargo, nuestro estudio presenta en forma detallada los recursos utilizados, permitiéndole a otros investigadores o tomadores de decisiones la asignación de los valores que crean apropiados para su institución.

La asignación de costos del tiempo perdido fue realizada de acuerdo al método del capital humano. Por este método, los costos indirectos se cuantifican en función de la ganancia perdida debido a la morbimortalidad producida para la enfermedad. Para cuantificar dicha pérdida se utilizó el salario mínimo vital y móvil. Esto podría resultar en una subestimación de dichos costos, al asignar el mínimo valor vigente en el mercado a la totalidad de los pacientes, dejando de lado profesiones y/o trabajos con mayores ingresos. Otros métodos alternativos de cuantificación de costos indirectos, como el método de costos de fricción, no pudieron ser calculados debido a falta de una fuente primaria para asignación de los mismos.

Por último, el bajo número de pacientes no permitió calcular márgenes precisos de costo-utilidad, pero sin embargo en el análisis de sensibilidad, ningún modelo se encontró por encima de los límites establecidos de disponibilidad a pagar. El bajo número se debió principalmente a los criterios estrictos de inclusión. Estos no permitían un reemplazo articular previo o enfermedad ósea-articular preexistente, siendo el principal motivo de los mismos una mayor homogeneidad de la muestra.

En conclusión, la osteoartritis de cadera y rodilla representa un deterioro multidimensional para la salud de los pacientes que la sufren. El reemplazo total articular es un procedimiento altamente efectivo para mejorar la calidad de vida y capacidad funcional de los mismos, con una razón de costo-utilidad aceptable tanto para la sociedad como para los prestadores de salud.

\section{BIBLIOGRAFÍA}

1. Becker R, Döring C, Denecke A, Brosz M. Expectation, satisfaction and clinical outcome of patients after total knee arthroplasty. Knee Surg Sports Traumatol Arthrosc. 2011;19(9):1433-1441.

2. Fortin PR, Clarke AE, Joseph L, et al. Outcomes of total hip and knee replacement: preoperative functional status predicts outcomes at six months after surgery. Arthritis Rheum. 1999;42(8):1722-1728.

3. Russo A, Elixhauser A, Steiner C, Wier L. HospitalBased Ambulatory Surgery, 2007. HEALTHCARE COST UTILIZATION PROJECT. 2010.

4. HCaU P. HCUP Facts and Figures: Statistics on HospitalBased Care in the United States, 2009. Healthcare Cost and Utilization Project. 2009.

5. Kurtz S, Ong K, Lau E, Mowat F, Halpern M. Projections of primary and revision hip and knee arthroplasty in the United States from 2005 to 2030. J Bone Joint Surg Am. 2007;89(4):780-785.

6. Losina E, Walensky RP, Kessler CL, et al. Cost-effectiveness of total knee arthroplasty in the United States: patient risk and hospital volume. Arch Intern Med. 2009;169(12):11131121; discussion 1121-1112.

7. Waimann CA, Fernandez-Mazarambroz RJ, Cantor SB, et al. Cost-effectiveness of total knee replacement: a prospective cohort study. Arthritis Care Res (Hoboken). 2014;66(4):592599.

8. Lopreite F, Garabano G, Harold S. Reemplazo total de cadera bilateral en un tiempo. Rev Asoc Argent Ortop Traumatol. 2011;76:146-151.

9. Mana Pastrián D, Garabano G, Nazur G, Pesciallo C, Vedoya S, Viale G. Reemplazo total de rodilla bilateral: Análisis de costos entre uno y dos tiempos. . Rev Asoc Argent Ortop Traumatol. 2010;75(2):151-156.

10. Plans IFoH. 2012 Comparative Price Report. Variation in Medical and Hospital Prices by Country 2012. Accessed 20 de Mayo, 2016.

11. Charlson ME, Pompei P, Ales KL, MacKenzie CR. A new method of classifying prognostic comorbidity in longitudinal studies: development and validation. J Chronic Dis. 1987;40(5):373-383.

12. Augustovski FA, Irazola VE, Velazquez AP, Gibbons L, Craig $\mathrm{BM}$. Argentine valuation of the EQ-5D health states. Value Health. 2009;12(4):587-596.

13. Knee Injury and Osteoarthritis outcome. Accessed 20 de Mayo, 2016.

14. Health NI. Patient Reported Outcomes Measurement Information System (PROMIS). Accessed 22 Mayo, 2016.

15. Drummond M, Sculpher M, Torrance G. Methods for the economic evaluation of health care programmes. Oxford University Press; 2005.

16. AMP. Actualización de los valores de IOMA. 2016. Accessed 22 de Mayo, 2016.

17. CADIME. NOMENCLADOR LABORATORIOS DE ANÁLISIS CLÍNICOS. 2016. Accessed 22 de Mayo, 2016.

18. ANMAT. (VNM) Vademecum Nacional de Medicamentos. 2016. Accessed 22 Mayo, 2016.

19. Resolución 4/2015. Salario Mínimo, Vital y Móvil Modificación. 2015. Accessed 22 Mayo, 2016.

20. Phibbs CS, Luft HS. Correlation of travel time on roads versus straight line distance. Med Care Res Rev. 1995;52(4):532-542.

21. CPIA. Costo por Kilómetro de un Automóvil Mediano para Uso Profesional a Febrero de 2016. 2016. Accessed 22 de Mayo, 2016.

22. ASSOCIATES R. Work Productivity and Activity Impairment Questionnaire (WPAI). 2016. Accessed 22 Mayo, 2016.

23. Ackerman IN, Bennell KL, Osborne RH. Decline in HealthRelated Quality of Life reported by more than half of those waiting for joint replacement surgery: a prospective cohort study. BMC Musculoskelet Disord. 2011;12:108.

24. Desmeules F, Dionne CE, Belzile E, Bourbonnais R, Frémont P. The burden of wait for knee replacement surgery: effects on pain, function and health-related quality of life at the time of surgery. Rheumatology (Oxford). 2010;49(5):945-954.

25. McHugh GA, Luker KA, Campbell M, Kay PR, Silman AJ. Pain, physical functioning and quality of life of individuals awaiting total joint replacement: a longitudinal study. J Eval Clin Pract. 2008;14(1):19-26.

26. FMI. World Economic and Financial Surveys. World Economic Outlook Database. 2016. Accessed 22 Mayo, 2016.

27. Escobar A, Quintana JM, Bilbao A, Aróstegui I, Lafuente I, Vidaurreta I. Responsiveness and clinically important differences for the WOMAC and SF-36 after total knee replacement. Osteoarthritis Cartilage. 2007;15(3):273-280. 